\title{
A New Inequality for Frames in Hilbert Spaces
}

\author{
Zhong-Qi Xiang $(\mathbb{D}$ \\ College of Mathematics and Computer Science, Shangrao Normal University, Shangrao, Jiangxi 334001, China
}

Correspondence should be addressed to Zhong-Qi Xiang; lxsy20110927@163.com

Received 15 July 2018; Accepted 20 September 2018; Published 3 October 2018

Academic Editor: Dashan Fan

Copyright (C) 2018 Zhong-Qi Xiang. This is an open access article distributed under the Creative Commons Attribution License, which permits unrestricted use, distribution, and reproduction in any medium, provided the original work is properly cited.

We obtain a new inequality for frames in Hilbert spaces associated with a scalar and a bounded linear operator induced by two Bessel sequences. It turns out that the corresponding results due to Balan et al. and Găvruţa can be deduced from our result.

\section{Introduction}

A frame for a Hilbert space firstly emerged in the work on nonharmonic Fourier series owing to Duffin and Schaeffer [1], which has made great contributions to various fields because of its nice properties; the reader can examine the papers [2-12] for background and details of frames.

Balan et al. in [13] showed us a surprising inequality when they further investigated the Parseval frame identity derived from their study on efficient algorithms for signal reconstruction, which was then extended to general frames and alternate dual frames by Găvruţa [14]. In this paper, we establish a new inequality for frames in Hilbert spaces, where a scalar and a bounded linear operator with respect to two Bessel sequences are involved, and it is shown that our result can lead to the corresponding results of Balan et al. and Găvruța.

The notations $\mathscr{H}, \mathrm{Id}_{\mathscr{H}}$, and $\sqrt{ }$ are reserved, respectively, for a complex Hilbert space, the identity operator on $\mathscr{H}$, and an index set which is finite or countable. The algebra of all bounded linear operators on $\mathscr{H}$ is designated as $B(\mathscr{H})$.

One says that a family $\left\{f_{j}\right\}_{j \in\rfloor}$ of vectors in $\mathscr{H}$ is a frame, if there are two positive constants $C, D>0$ satisfying

$$
C\|f\|^{2} \leq \sum_{j \in \rrbracket}\left|\left\langle f, f_{j}\right\rangle\right|^{2} \leq D\|f\|^{2}, \quad \forall f \in \mathscr{H} .
$$

The frame $\left\{f_{j}\right\}_{j \in\rfloor}$ is said to be Parseval if $C=D=1$. If $\left\{f_{j}\right\}_{j \in \rrbracket}$ satisfies the inequality to the right in (1), we call that $\left\{f_{j}\right\}_{j \in \rrbracket}$ is a Bessel sequence for $\mathscr{H}$.
For a given frame $\mathscr{F}=\left\{f_{j}\right\}_{j \in \rrbracket}$, the frame operator $S_{\mathscr{F}}$, a positive, self-adjoint, and invertible operator on $\mathscr{H}$, is defined by

$$
\begin{aligned}
S_{\mathscr{F}}: \mathscr{H} & \longrightarrow \mathscr{H}, \\
S_{\mathscr{F}} f & =\sum_{j \in \rrbracket}\left\langle f, f_{j}\right\rangle f_{j},
\end{aligned}
$$

$$
\forall f \in \mathscr{H},
$$

from which we see that

$$
f=\sum_{j \in \unlhd}\left\langle f, f_{j}\right\rangle S_{\mathscr{F}}^{-1} f_{j}=\sum_{j \in \unlhd}\left\langle f, S_{\mathscr{F}}^{-1} f_{j}\right\rangle f_{j}, \quad \forall f \in \mathscr{H},
$$

where the involved frame $\left\{\tilde{f}_{j}=S_{\mathscr{F}}^{-1} f_{j}\right\}_{j \in \rrbracket}$ is said to be the canonical dual of $\left\{f_{j}\right\}_{j \in \Downarrow}$.

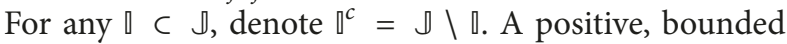
linear, and self-adjoint operator induced by $\llbracket$ and the frame $\mathscr{F}=\left\{f_{j}\right\}_{j \in\rfloor}$ is given below

$$
\begin{aligned}
S_{\square}^{\mathscr{F}}: \mathscr{H} & \longrightarrow \mathscr{H}, \\
S_{\square}^{\mathscr{F}} f & =\sum_{j \in \rrbracket}\left\langle f, f_{j}\right\rangle f_{j},
\end{aligned}
$$

$$
\forall f \in \mathscr{H} .
$$

Suppose that $\mathscr{F}=\left\{f_{j}\right\}_{j \in \rrbracket}$ and $\mathscr{G}=\left\{g_{j}\right\}_{j \in \rrbracket}$ are two Bessel sequences for $\mathscr{H}$. An application of the CauchySchwartzinequality can show that the operator 


$$
\begin{aligned}
S_{\mathscr{F} \mathscr{G}}: \mathscr{H} & \longrightarrow \mathscr{H}, \\
S_{\mathscr{F} \mathscr{G}} f & =\sum_{j \in \rrbracket}\left\langle f, g_{j}\right\rangle f_{j}
\end{aligned}
$$

is well-defined and further $S_{\mathscr{F} \mathscr{G}} \in B(\mathscr{H})$. Particularly, if $S_{\mathscr{F} \mathscr{G}}=\operatorname{Id}_{\mathscr{H}}$, then both $\left\{f_{j}\right\}_{j \in \rrbracket}$ and $\left\{g_{j}\right\}_{j \in \rrbracket}$ are frames for $\mathscr{H}$. In this case we say that $\left\{g_{j}\right\}_{j \in \rrbracket}$ is an alternate dual frame of $\left\{f_{j}\right\}_{j \in \rrbracket}$, and the pair $\left(\left\{f_{j}\right\}_{j \in \rrbracket},\left\{g_{j}\right\}_{j \in \rrbracket}\right)$ is called an alternate dual frame pair.

\section{The Main Results}

We need the following simple result on operators to present our main result.

Lemma 1. Suppose that $U, V, L \in B(\mathscr{H})$ and that $U+V=L$. Then for each $\lambda \in[0,1]$ we have

$$
\begin{aligned}
& U^{*} U+\lambda\left(V^{*} L+L^{*} V\right) \\
& =V^{*} V+(1-\lambda)\left(U^{*} L+L^{*} U\right)+(2 \lambda-1) L^{*} L \\
& \geq\left(2 \lambda-\lambda^{2}\right) L^{*} L .
\end{aligned}
$$

Proof. A direct calculation gives

$$
\begin{array}{rl}
U^{*} & U+\lambda\left(V^{*} L+L^{*} V\right) \\
& =U^{*} U+\lambda\left(\left(L^{*}-U^{*}\right) L+L^{*}(L-U)\right) \\
& =U^{*} U+\lambda\left(L^{*} L-U^{*} L+L^{*} L-L^{*} U\right) \\
& =U^{*} U-\lambda\left(U^{*} L+L^{*} U\right)+2 \lambda L^{*} L .
\end{array}
$$

From this fact and taking into account that

$$
\begin{aligned}
V^{*} V+ & (1-\lambda)\left(U^{*} L+L^{*} U\right)+(2 \lambda-1) L^{*} L \\
= & \left(L^{*}-U^{*}\right)(L-U)+(1-\lambda)\left(U^{*} L+L^{*} U\right) \\
& +(2 \lambda-1) L^{*} L \\
= & L^{*} L-\left(L^{*} U+U^{*} L\right)+U^{*} U \\
& +(1-\lambda)\left(U^{*} L+L^{*} U\right)+(2 \lambda-1) L^{*} L \\
= & U^{*} U-\lambda\left(U^{*} L+L^{*} U\right)+2 \lambda L^{*} L \\
= & (U-\lambda L)^{*}(U-\lambda L)+\left(2 \lambda-\lambda^{2}\right) L^{*} L \\
\geq & \left(2 \lambda-\lambda^{2}\right) L^{*} L,
\end{aligned}
$$

we arrive at the relation stated in the lemma.

We can immediately get the following result obtained by Poria in [15], when putting $L=\operatorname{Id}_{\mathscr{H}}$ in Lemma 1.

Corollary 2. Suppose that $U, V \in B(\mathscr{H})$ and that $U+V=$ $\operatorname{Id}_{\mathscr{H}}$. Then for every $\lambda \in[0,1]$ we have

$$
\begin{aligned}
U^{*} U+\lambda\left(V^{*}+V\right)= & V^{*} V+(1-\lambda)\left(U^{*}+U\right) \\
& +(2 \lambda-1) \operatorname{Id}_{\mathscr{H}} \\
\geq & \left(2 \lambda-\lambda^{2}\right) \operatorname{Id}_{\mathscr{H}} .
\end{aligned}
$$

Theorem 3. Suppose that $\left\{f_{j}\right\}_{j \in \rrbracket}$ is a frame for $\mathscr{H}$, that $\left\{g_{j}\right\}_{j \in \rrbracket}$ and $\left\{h_{j}\right\}_{j \in \rrbracket}$ are two Bessel sequences for $\mathscr{H}$, and that the operator $S_{\mathscr{F} G}$ is defined by (5). Then for each $\lambda \in[0,1]$ and each $f \in \mathscr{H}$, we have

$$
\begin{gathered}
\left\|\sum_{j \in \rrbracket}\left\langle f, g_{j}-h_{j}\right\rangle f_{j}\right\|^{2}+\operatorname{Re} \sum_{j \in \rrbracket}\left\langle f, h_{j}\right\rangle\left\langle f_{j}, S_{\mathscr{F} \mathscr{G}} f\right\rangle \\
=\left\|\sum_{j \in \rrbracket}\left\langle f, h_{j}\right\rangle f_{j}\right\|^{2} \\
\quad+\operatorname{Re} \sum_{j \in \rrbracket}\left\langle f, g_{j}-h_{j}\right\rangle\left\langle f_{j}, S_{\mathscr{F} \mathscr{G}} f\right\rangle \\
\geq\left(2 \lambda-\lambda^{2}\right) \operatorname{Re} \sum_{j \in \rrbracket}\left\langle f, g_{j}-h_{j}\right\rangle\left\langle f_{j}, S_{\mathscr{F} \mathscr{G}} f\right\rangle \\
+\left(1-\lambda^{2}\right) \operatorname{Re} \sum_{j \in \rrbracket}\left\langle f, h_{j}\right\rangle\left\langle f_{j}, S_{\mathscr{F} \mathscr{G}} f\right\rangle .
\end{gathered}
$$

Moreover, if $S_{\mathscr{F} \mathscr{G}}$ is self-adjoint, then for any $\lambda \in[0,1]$ and any $f \in \mathscr{H}$,

$$
\begin{aligned}
& \left\|\sum_{j \in \rrbracket}\left\langle f, f_{j}\right\rangle\left(g_{j}-h_{j}\right)\right\|^{2}+\operatorname{Re} \sum_{j \in \rrbracket}\left\langle f, S_{\mathscr{F} \mathscr{G}} h_{j}\right\rangle\left\langle f_{j}, f\right\rangle \\
& =\left\|\sum_{j \in \rrbracket}\left\langle f, f_{j}\right\rangle h_{j}\right\|^{2} \\
& \quad+\operatorname{Re} \sum_{j \in \rrbracket}\left\langle f, S_{\mathscr{F} \mathscr{G}}\left(g_{j}-h_{j}\right)\right\rangle\left\langle f_{j}, f\right\rangle \\
& \geq\left(2 \lambda-\lambda^{2}\right) \operatorname{Re} \sum_{j \in \rrbracket}\left\langle f, S_{\mathscr{F} \mathscr{G}}\left(g_{j}-h_{j}\right)\right\rangle\left\langle f_{j}, f\right\rangle \\
& \quad+\left(1-\lambda^{2}\right) \operatorname{Re} \sum_{j \in \rrbracket}\left\langle f, S_{\mathscr{F} \mathscr{G}} h_{j}\right\rangle\left\langle f_{j}, f\right\rangle .
\end{aligned}
$$

Proof. We take Uf $=\sum_{j \in \rrbracket}\left\langle f, g_{j}-h_{j}\right\rangle f_{j}$ and $V f=$ $\sum_{j \in \mathbb{J}}\left\langle f, h_{j}\right\rangle f_{j}$ for any $f \in \mathscr{H}$. Then $U, V \in B(\mathscr{H})$ and further

$$
\begin{aligned}
U f+V f & =\sum_{j \in \rrbracket}\left\langle f, g_{j}-h_{j}\right\rangle f_{j}+\sum_{j \in \rrbracket}\left\langle f, h_{j}\right\rangle f_{j} \\
& =\sum_{j \in \rrbracket}\left\langle f, g_{j}\right\rangle f_{j}=S_{\mathscr{F} \mathscr{G}} f .
\end{aligned}
$$

By Lemma 1 we have

$$
\begin{aligned}
\|U f\|^{2} & +2 \lambda \operatorname{Re}\left\langle S_{\mathscr{F} \mathscr{G}}^{*} V f, f\right\rangle \\
= & \|V f\|^{2}+2(1-\lambda) \operatorname{Re}\left\langle S_{\mathscr{F} \mathscr{G}}^{*} U f, f\right\rangle \\
& +(2 \lambda-1) \operatorname{Re}\left\langle S_{\mathscr{F} \mathscr{G}} f, S_{\mathscr{F} \mathscr{G}} f\right\rangle .
\end{aligned}
$$


Therefore,

$$
\begin{aligned}
\|U f\|^{2}= & \|V f\|^{2}+2(1-\lambda) \operatorname{Re}\left\langle S_{\mathscr{F} \mathscr{G}}^{*} U f, f\right\rangle \\
& +(2 \lambda-1) \operatorname{Re}\left\langle S_{\mathscr{F} \mathscr{G}} f, S_{\mathscr{F} \mathscr{G}} f\right\rangle \\
& -2 \lambda \operatorname{Re}\left\langle S_{\mathscr{F} \mathscr{G}}^{*} V f, f\right\rangle \\
= & \|V f\|^{2}+2 \operatorname{Re}\left\langle S_{\mathscr{F} \mathscr{G}}^{*} U f, f\right\rangle \\
& -2 \lambda \operatorname{Re}\left\langle\left(S_{\mathscr{F} \mathscr{G}}^{*} U+S_{\mathscr{F} \mathscr{G}}^{*} V\right) f, f\right\rangle \\
& +(2 \lambda-1) \operatorname{Re}\left\langle S_{\mathscr{F} \mathscr{G}} f, S_{\mathscr{F} \mathscr{G}} f\right\rangle \\
= & \|V f\|^{2}+2 \operatorname{Re}\left\langle S_{\mathscr{F} \mathscr{G}}^{*} U f, f\right\rangle \\
& -\operatorname{Re}\left\langle S_{\mathscr{F} \mathscr{G}} f, S_{\mathscr{F} \mathscr{G}} f\right\rangle \\
= & \|V f\|^{2}+2 \operatorname{Re}\left\langle S_{\mathscr{F} \mathscr{G}}^{*} U f, f\right\rangle \\
& -\operatorname{Re}\left\langle S_{\mathscr{F} \mathscr{G}}^{*}(U+V) f, f\right\rangle \\
= & \|V f\|^{2}+\operatorname{Re}\left\langle S_{\mathscr{F} \mathscr{G}}^{*} U f, f\right\rangle-\operatorname{Re}\left\langle S_{\mathscr{F} \mathscr{G}}^{*} V f, f\right\rangle .
\end{aligned}
$$

It follows that

$$
\begin{aligned}
\left\|\sum_{j \in \rrbracket}\left\langle f, g_{j}-h_{j}\right\rangle f_{j}\right\|^{2}+\operatorname{Re} \sum_{j \in \unlhd}\left\langle f, h_{j}\right\rangle\left\langle f_{j}, S_{\mathscr{F} \mathscr{G}} f\right\rangle \\
=\|U f\|^{2}+\operatorname{Re}\left\langle S_{\mathscr{F} \mathscr{G}}^{*} V f, f\right\rangle \\
=\|V f\|^{2}+\operatorname{Re}\left\langle S_{\mathscr{F} \mathscr{G}}^{*} U f, f\right\rangle \\
=\left\|\sum_{j \in \unlhd}\left\langle f, h_{j}\right\rangle f_{j}\right\|^{2} \\
\quad+\operatorname{Re} \sum_{j \in \rrbracket}\left\langle f, g_{j}-h_{j}\right\rangle\left\langle f_{j}, S_{\mathscr{F} \mathscr{G}} f\right\rangle .
\end{aligned}
$$

We now prove the inequality in (10). Again by Lemma 1 ,

$$
\begin{aligned}
& \|U f\|^{2}+2 \lambda \operatorname{Re}\left\langle S_{\mathscr{F} \mathscr{G}}^{*} V f, f\right\rangle \\
& \geq\left(2 \lambda-\lambda^{2}\right)\left\langle S_{\mathscr{F} \mathscr{G}}^{*} S_{\mathscr{F} \mathscr{G}} f, f\right\rangle
\end{aligned}
$$

for every $f \in \mathscr{H}$. Hence,

$$
\begin{aligned}
\|U f\|^{2} \geq & \left(2 \lambda-\lambda^{2}\right)\left\langle S_{\mathscr{F} \mathscr{G}}^{*} S_{\mathscr{F} \mathscr{G}} f, f\right\rangle \\
& -2 \lambda \operatorname{Re}\left\langle S_{\mathscr{F} \mathscr{G}}^{*} V f, f\right\rangle \\
= & \left(2 \lambda-\lambda^{2}\right)\left\langle S_{\mathscr{F} \mathscr{G}} f, S_{\mathscr{F} \mathscr{G}} f\right\rangle \\
& -2 \lambda \operatorname{Re}\left\langle V f, S_{\mathscr{F} \mathscr{G}} f\right\rangle \\
= & \left(2 \lambda-\lambda^{2}\right) \operatorname{Re}\left\langle(U+V) f, S_{\mathscr{F} \mathscr{G}} f\right\rangle \\
& -2 \lambda \operatorname{Re}\left\langle V f, S_{\mathscr{F} \mathscr{G}} f\right\rangle \\
= & \left(2 \lambda-\lambda^{2}\right) \operatorname{Re}\left\langle U f, S_{\mathscr{F} \mathscr{G}} f\right\rangle \\
& -\lambda^{2} \operatorname{Re}\left\langle V f, S_{\mathscr{F} \mathscr{G}} f\right\rangle,
\end{aligned}
$$

from which we conclude that

$$
\begin{aligned}
&\left\|\sum_{j \in \unlhd}\left\langle f, g_{j}-h_{j}\right\rangle f_{j}\right\|^{2}+\operatorname{Re} \sum_{j \in \unlhd}\left\langle f, h_{j}\right\rangle\left\langle f_{j}, S_{\mathscr{F} \mathscr{G}} f\right\rangle \\
&=\|U f\|^{2}+\operatorname{Re}\left\langle V f, S_{\mathscr{F} \mathscr{G}} f\right\rangle \\
& \geq\left(2 \lambda-\lambda^{2}\right) \operatorname{Re}\left\langle U f, S_{\mathscr{F} \mathscr{G}} f\right\rangle \\
&+\left(1-\lambda^{2}\right) \operatorname{Re}\left\langle V f, S_{\mathscr{F} \mathscr{G}} f\right\rangle \\
&=\left(2 \lambda-\lambda^{2}\right) \operatorname{Re} \sum_{j \in \unlhd}\left\langle f, g_{j}-h_{j}\right\rangle\left\langle f_{j}, S_{\mathscr{F} \mathscr{G}} f\right\rangle \\
&+\left(1-\lambda^{2}\right) \operatorname{Re} \sum_{j \in \unlhd}\left\langle f, h_{j}\right\rangle\left\langle f_{j}, S_{\mathscr{F} \mathscr{G}} f\right\rangle .
\end{aligned}
$$

The proof of (11) is similar to the proof of (10); we leave the details to the reader.

Corollary 4. Suppose that $\left\{f_{j}\right\}_{j \in \rrbracket}$ is a frame for $\mathscr{H}$ with frame operator $S_{\mathscr{F}}$ and that $\tilde{f}_{j}=S_{\mathscr{F}}^{-1} f_{j}$ for any $j \in \mathbb{J}$. Then for all $\lambda \in[0,1]$, for any $\llbracket \subset \rrbracket$ and any $f \in \mathscr{H}$, we have

$$
\begin{aligned}
& \sum_{j \in \mathbb{\mathbb { V }}^{c}}\left|\left\langle f, f_{j}\right\rangle\right|^{2}+\sum_{j \in \rrbracket}\left|\left\langle S_{\square}^{\mathscr{F}} f, \tilde{f}_{j}\right\rangle\right|^{2} \\
& =\sum_{j \in \mathbb{}}\left|\left\langle f, f_{j}\right\rangle\right|^{2}+\sum_{j \in \rrbracket}\left|\left\langle S_{\mathbb{u}^{c}}^{\mathscr{F}} f, \tilde{f}_{j}\right\rangle\right|^{2} \\
& \geq\left(2 \lambda-\lambda^{2}\right) \sum_{j \in \mathbb{1}}\left|\left\langle f, f_{j}\right\rangle\right|^{2} \\
& +\left(1-\lambda^{2}\right) \sum_{j \in \mathbb{c}^{c}}\left|\left\langle f, f_{j}\right\rangle\right|^{2} .
\end{aligned}
$$

Proof. Setting $g_{j}=S_{\mathscr{F}}^{-1 / 2} f_{j}$ for each $j \in \mathbb{J}$, then $S_{\mathscr{F} \mathscr{G}}=S_{\mathscr{F}}^{1 / 2}$. Taking

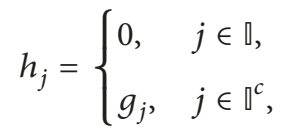

then $\left\{g_{j}\right\}_{j \in \rrbracket}$ and $\left\{h_{j}\right\}_{j \in \rrbracket}$ are both Bessel sequences for $\mathscr{H}$. For any $f \in \mathscr{H}$ we have

$$
\begin{aligned}
& \left\|\sum_{j \in \rrbracket}\left\langle f, f_{j}\right\rangle\left(g_{j}-h_{j}\right)\right\|^{2}=\left\|\sum_{j \in \mathbb{U}}\left\langle f, f_{j}\right\rangle S_{\mathscr{F}}^{-1 / 2} f_{j}\right\|^{2} \\
& =\left\|S_{\mathscr{F}}^{-1 / 2} S_{\square}^{\mathscr{F}} f\right\|^{2}=\left\langle S_{\mathscr{F}}^{-1 / 2} S_{\square}^{\mathscr{F}} f, S_{\mathscr{F}}^{-1 / 2} S_{\square}^{\mathscr{F}} f\right\rangle \\
& =\left\langle S_{\square}^{\mathscr{F}} f, S_{\mathscr{F}}^{-1} S_{\square}^{\mathscr{F}} f\right\rangle=\left\langle S_{\mathscr{F}} S_{\mathscr{F}}^{-1} S_{\square}^{\mathscr{F}} f, S_{\mathscr{F}}^{-1} S_{\square}^{\mathscr{F}} f\right\rangle \\
& =\sum_{j \in \unlhd}\left\langle S_{\mathscr{F}}^{-1} S_{\square}^{\mathscr{F}} f, f_{j}\right\rangle\left\langle f_{j}, S_{\mathscr{F}}^{-1} S_{\square}^{\mathscr{F}} f\right\rangle \\
& =\sum_{j \in \unlhd}\left\langle S_{\square}^{\mathscr{F}} f, S_{\mathscr{F}}^{-1} f_{j}\right\rangle\left\langle S_{\mathscr{F}}^{-1} f_{j}, S_{\square}^{\mathscr{F}} f\right\rangle \\
& =\sum_{j \in \unlhd}\left|\left\langle S_{\square}^{\mathscr{F}} f, \tilde{f}_{j}\right\rangle\right|^{2} .
\end{aligned}
$$


A similar discussion yields

$$
\left\|\sum_{j \in \rrbracket}\left\langle f, f_{j}\right\rangle h_{j}\right\|^{2}=\sum_{j \in \rrbracket}\left|\left\langle S_{a^{c}}^{\mathscr{F}} f, \tilde{f}_{j}\right\rangle\right|^{2} .
$$

We also have

$$
\begin{array}{r}
\operatorname{Re} \sum_{j \in \rrbracket}\left\langle f, S_{\mathscr{F} \mathscr{G}} h_{j}\right\rangle\left\langle f_{j}, f\right\rangle=\sum_{j \in \mathbb{\natural}^{c}}\left|\left\langle f, f_{j}\right\rangle\right|^{2}, \\
\operatorname{Re} \sum_{j \in \rrbracket}\left\langle f, S_{\mathscr{F} \mathscr{G}}\left(g_{j}-h_{j}\right)\right\rangle\left\langle f_{j}, f\right\rangle=\sum_{j \in \rrbracket}\left|\left\langle f, f_{j}\right\rangle\right|^{2} .
\end{array}
$$

Thus the result follows from Theorem 3.

Let $\left\{f_{j}\right\}_{j \in \rrbracket}$ be a Parseval frame for $\mathscr{H}$; then $S_{\mathscr{F}}=\operatorname{Id}_{\mathscr{H}}$. Thus for any $₫ \subset \rrbracket$,

$$
\begin{aligned}
\sum_{j \in \rrbracket}\left|\left\langle S_{\square}^{\mathscr{F}} f, \tilde{f}_{j}\right\rangle\right|^{2} & =\sum_{j \in \rrbracket}\left|\left\langle S_{\square}^{\mathscr{F}} f, f_{j}\right\rangle\right|^{2}=\left\|S_{\square}^{\mathscr{F}} f\right\|^{2} \\
& =\left\|\sum_{j \in \rrbracket}\left\langle f, f_{j}\right\rangle f_{j}\right\|^{2}, \quad \forall f \in \mathscr{H} .
\end{aligned}
$$

Similarly we have

$$
\sum_{j \in \rrbracket}\left|\left\langle S_{\mathbb{C}^{c}}^{\mathscr{F}} f, \tilde{f}_{j}\right\rangle\right|^{2}=\left\|\sum_{j \in \mathbb{I}^{c}}\left\langle f, f_{j}\right\rangle f_{j}\right\|^{2} .
$$

This together with Corollary 4 leads to a result as follows.

Corollary 5. Suppose that $\left\{f_{j}\right\}_{j \in \rrbracket}$ is a Parseval frame for $\mathscr{H}$. Then for each $\lambda \in[0,1]$, for any $\subset \mathbb{I}$ and any $f \in \mathscr{H}$, we have

$$
\begin{aligned}
& \sum_{j \in \mathbb{\rrbracket}^{c}}\left|\left\langle f, f_{j}\right\rangle\right|^{2}+\left\|\sum_{j \in \mathbb{D}}\left\langle f, f_{j}\right\rangle f_{j}\right\|^{2} \\
& =\sum_{j \in \mathbb{1}}\left|\left\langle f, f_{j}\right\rangle\right|^{2}+\left\|\sum_{j \in \mathbb{1}^{c}}\left\langle f, f_{j}\right\rangle f_{j}\right\|^{2} \\
& \geq\left(2 \lambda-\lambda^{2}\right) \sum_{j \in \mathbb{}}\left|\left\langle f, f_{j}\right\rangle\right|^{2} \\
& +\left(1-\lambda^{2}\right) \sum_{j \in \mathbb{I}^{c}}\left|\left\langle f, f_{j}\right\rangle\right|^{2} .
\end{aligned}
$$

Corollary 6. Suppose that $\left(\left\{f_{j}\right\}_{j \in \rrbracket},\left\{g_{j}\right\}_{j \in \rrbracket}\right)$ is an alternate dual frame pair for $\mathscr{H}$. Then for each $\lambda \in[0,1]$, for any $\square \subset \mathbb{}$ and any $f \in \mathscr{H}$, we have

$$
\begin{aligned}
& \left\|\sum_{j \in \mathbb{\complement}}\left\langle f, g_{j}\right\rangle f_{j}\right\|^{2}+\operatorname{Re} \sum_{j \in \mathbb{I}^{c}}\left\langle f, g_{j}\right\rangle\left\langle f_{j}, f\right\rangle \\
& =\left\|\sum_{j \in \mathbb{1}^{c}}\left\langle f, g_{j}\right\rangle f_{j}\right\|^{2}+\operatorname{Re} \sum_{j \in \mathbb{D}}\left\langle f, g_{j}\right\rangle\left\langle f_{j}, f\right\rangle \\
& \geq\left(2 \lambda-\lambda^{2}\right) \operatorname{Re} \sum_{j \in \rrbracket}\left\langle f, g_{j}\right\rangle\left\langle f_{j}, f\right\rangle \\
& +\left(1-\lambda^{2}\right) \operatorname{Re} \sum_{j \in \mathbb{1}^{c}}\left\langle f, g_{j}\right\rangle\left\langle f_{j}, f\right\rangle .
\end{aligned}
$$

Proof. Since $\left\{g_{j}\right\}_{j \in \rrbracket}$ is an alternate dual frame of $\left\{f_{j}\right\}_{j \in \rrbracket}$, $S_{\mathscr{F} \mathscr{G}}=\operatorname{Id}_{\mathscr{H}}$. For any $j \in \mathbb{J}$, let

$$
h_{j}= \begin{cases}0, & j \in \mathbb{\mathbb { V }}, \\ g_{j}, & j \in \mathbb{\mathbb { V }}^{\mathrm{c}} .\end{cases}
$$

On the one hand we have

$$
\begin{aligned}
& \left\|\sum_{j \in \rrbracket}\left\langle f, g_{j}-h_{j}\right\rangle f_{j}\right\|^{2}=\left\|\sum_{j \in \rrbracket}\left\langle f, g_{j}\right\rangle f_{j}\right\|^{2}, \\
& \left\|\sum_{j \in \rrbracket}\left\langle f, h_{j}\right\rangle f_{j}\right\|^{2}=\left\|\sum_{j \in \mathbb{I}^{c}}\left\langle f, g_{j}\right\rangle f_{j}\right\|^{2} .
\end{aligned}
$$

On the other hand we have

$$
\begin{aligned}
& \operatorname{Re} \sum_{j \in \rrbracket}\left\langle f, h_{j}\right\rangle\left\langle f_{j}, S_{\mathscr{F} \mathscr{G}} f\right\rangle=\operatorname{Re} \sum_{j \in \mathbb{C}^{c}}\left\langle f, g_{j}\right\rangle\left\langle f_{j}, f\right\rangle, \\
& \operatorname{Re} \sum_{j \in \rrbracket}\left\langle f, g_{j}-h_{j}\right\rangle\left\langle f_{j}, S_{\mathscr{F} \mathscr{G}} f\right\rangle \\
& \quad=\operatorname{Re} \sum_{j \in \mathbb{N}}\left\langle f, g_{j}\right\rangle\left\langle f_{j}, f\right\rangle .
\end{aligned}
$$

By Theorem 3 the conclusion follows.

Remark 7. Theorems 2.2 and 3.2 in [14] and Proposition 4.1 in [13] can be obtained when taking $\lambda=1 / 2$, respectively, in Corollaries 4, 6, and 5 .

As a matter of fact, we can establish a more general inequality for alternate dual frames than that shown in Corollary 6. 
Theorem 8. Suppose that $\left(\left\{f_{j}\right\}_{j \in \rrbracket},\left\{g_{j}\right\}_{j \in \rrbracket}\right)$ is an alternate dual frame pair for $\mathscr{H}$. Then for every bounded sequence $\left\{\omega_{j}\right\}_{j \in \rrbracket}$, for all $\lambda \in[0,1]$ and all $f \in \mathscr{H}$, we have

$$
\begin{aligned}
& \operatorname{Re} \sum_{j \in \rrbracket} \omega_{j}\left\langle f, g_{j}\right\rangle\left\langle f_{j}, f\right\rangle+\left\|\sum_{j \in \rrbracket}\left(1-\omega_{j}\right)\left\langle f, g_{j}\right\rangle f_{j}\right\|^{2} \\
& \geq\left(2 \lambda-\lambda^{2}\right) \operatorname{Re} \sum_{j \in \rrbracket}\left(1-\omega_{j}\right)\left\langle f, g_{j}\right\rangle\left\langle f_{j}, f\right\rangle \\
& \quad+\left(1-\lambda^{2}\right) \operatorname{Re} \sum_{j \in \rrbracket} \omega_{j}\left\langle f, g_{j}\right\rangle\left\langle f_{j}, f\right\rangle .
\end{aligned}
$$

Proof. We define the operators $F_{\omega}$ and $F_{1-\omega}$ by

$$
\begin{aligned}
F_{\omega} f & =\sum_{j \in \rrbracket} \omega_{j}\left\langle f, g_{j}\right\rangle f_{j}, \\
F_{1-\omega} f & =\sum_{j \in \rrbracket}\left(1-\omega_{j}\right)\left\langle f, g_{j}\right\rangle f_{j} .
\end{aligned}
$$

Then both series converge unconditionally and $F_{\omega}, F_{1-\omega} \in$ $B(\mathscr{H})$. Since $F_{\omega}+F_{1-\omega}=\operatorname{Id}_{\mathscr{H}}$, by Corollary 2 we obtain

$$
\begin{aligned}
& \left\langle F_{1-\omega}^{*} F_{1-\omega} f, f\right\rangle+\lambda \overline{\left\langle F_{\omega} f, f\right\rangle}+\lambda\left\langle F_{\omega} f, f\right\rangle \\
& \quad \geq\left(2 \lambda-\lambda^{2}\right)\|f\|^{2}
\end{aligned}
$$

for each $f \in \mathscr{H}$. Hence

$$
\left\|F_{1-\omega} f\right\|^{2}+2 \lambda \operatorname{Re}\left\langle F_{\omega} f, f\right\rangle \geq\left(2 \lambda-\lambda^{2}\right)\langle f, f\rangle .
$$

Therefore,

$$
\begin{aligned}
\left\|F_{1-\omega} f\right\|^{2} \geq & \left(2 \lambda-\lambda^{2}\right)\langle f, f\rangle-2 \lambda \operatorname{Re}\left\langle F_{\omega} f, f\right\rangle \\
= & \left(2 \lambda-\lambda^{2}\right) \operatorname{Re}\left\langle\left(F_{\omega}+F_{1-\omega}\right) f, f\right\rangle \\
& -2 \lambda \operatorname{Re}\left\langle F_{\omega} f, f\right\rangle \\
= & \left(2 \lambda-\lambda^{2}\right) \operatorname{Re}\left\langle F_{1-\omega} f, f\right\rangle \\
& -\lambda^{2} \operatorname{Re}\left\langle F_{\omega} f, f\right\rangle .
\end{aligned}
$$

It follows that

$$
\begin{aligned}
\operatorname{Re} & \sum_{j \in \rrbracket} \omega_{j}\left\langle f, g_{j}\right\rangle\left\langle f_{j}, f\right\rangle+\left\|\sum_{j \in \rrbracket}\left(1-\omega_{j}\right)\left\langle f, g_{j}\right\rangle f_{j}\right\|^{2} \\
= & \operatorname{Re}\left\langle F_{\omega} f, f\right\rangle+\left\|F_{1-\omega} f\right\|^{2} \\
\geq & \left(2 \lambda-\lambda^{2}\right) \operatorname{Re}\left\langle F_{1-\omega} f, f\right\rangle \\
& +\left(1-\lambda^{2}\right) \operatorname{Re}\left\langle F_{\omega} f, f\right\rangle \\
= & \left(2 \lambda-\lambda^{2}\right) \operatorname{Re} \sum_{j \in \rrbracket}\left(1-\omega_{j}\right)\left\langle f, g_{j}\right\rangle\left\langle f_{j}, f\right\rangle \\
& +\left(1-\lambda^{2}\right) \operatorname{Re} \sum_{j \in \rrbracket} \omega_{j}\left\langle f, g_{j}\right\rangle\left\langle f_{j}, f\right\rangle .
\end{aligned}
$$

This completes the proof.
Remark 9. If we take $\lambda=1 / 2$ in Theorem 8 , then we can obtain Theorem 3.3 in [14].

\section{Data Availability}

No data were used to support this study.

\section{Conflicts of Interest}

The author declares that he has no conflicts of interest.

\section{Acknowledgments}

This work is supported by the National Natural Science Foundation of China (Grant Nos. 11761057 and 11561057).

\section{References}

[1] R. J. Duffin and A. C. Schaeffer, "A class of nonharmonic Fourier series," Transactions of the American Mathematical Society, vol. 72, pp. 341-366, 1952.

[2] J. J. Benedetto, A. M. Powell, and O. Yilmaz, "Sigma-Delta $(\Sigma \Delta)$ quantization and finite frames," IEEE Transactions on Information Theory, vol. 52, no. 5, pp. 1990-2005, 2006.

[3] P. G. Casazza, “The art of frame theory," Taiwanese Journal of Mathematics, vol. 4, no. 2, pp. 129-201, 2000.

[4] O. Christensen, An Introduction to Frames and Riesz Bases, Applied and Numerical Harmonic Analysis, Birkhäuser, Boston, Mass, USA, 2003.

[5] Z. Cvetkovic and M. Vetterli, “Oversampled filter banks," IEEE Transactions on Signal Processing, vol. 46, no. 5, pp. 1245-1255, 1998.

[6] I. Daubechies, A. Grossmann, and Y. Meyer, "Painless nonorthogonal expansions," Journal of Mathematical Physics, vol. 27, no. 5, pp. 1271-1283, 1986.

[7] Y. C. Eldar and J. Forney, "Optimal tight frames and quantum measurement," Institute of Electrical and Electronics Engineers Transactions on Information Theory, vol. 48, no. 3, pp. 599-610, 2002.

[8] H. G. Feichtinger and K. Gröchenig, "Theory and practice of irregular sampling," in Wavelets: mathematics and applications, Stud. Adv. Math., pp. 305-363, CRC, Boca Raton, FL, USA, 1994.

[9] D. Han and D. R. Larson, "Frames, bases and group representations," Memoirs of the American Mathematical Society, vol. 147, no. $697,2000$.

[10] D. Han and W. Sun, "Reconstruction of signals from frame coefficients with erasures at unknown locations," Institute of Electrical and Electronics Engineers Transactions on Information Theory, vol. 60, no. 7, pp. 4013-4025, 2014.

[11] T. Strohmer and J. Heath, "Grassmannian frames with applications to coding and communication," Applied and Computational Harmonic Analysis, vol. 14, no. 3, pp. 257-275, 2003.

[12] W. Sun, "Asymptotic properties of Gabor frame operators as sampling density tends to infinity," Journal of Functional Analysis, vol. 258, no. 3, pp. 913-932, 2010.

[13] R. Balan, P. G. Casazza, D. Edidin, and G. Kutyniok, "A new identity for Parseval frames," Proceedings of the American Mathematical Society, vol. 135, no. 4, pp. 1007-1015, 2007. 
[14] P. Gavruta, "On some identities and inequalities for frames in Hilbert spaces," Journal of Mathematical Analysis and Applications, vol. 321, no. 1, pp. 469-478, 2006.

[15] A. Poria, "Some identities and inequalities for Hilbert-Schmidt frames," Mediterranean Journal of Mathematics, vol. 14, no. 2, Art. 59, 14 pages, 2017. 


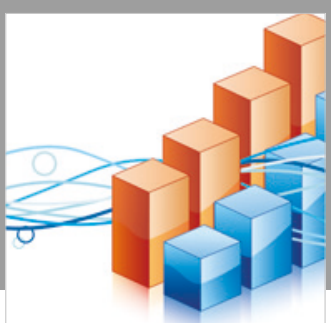

Advances in

Operations Research

\section{-n-m}
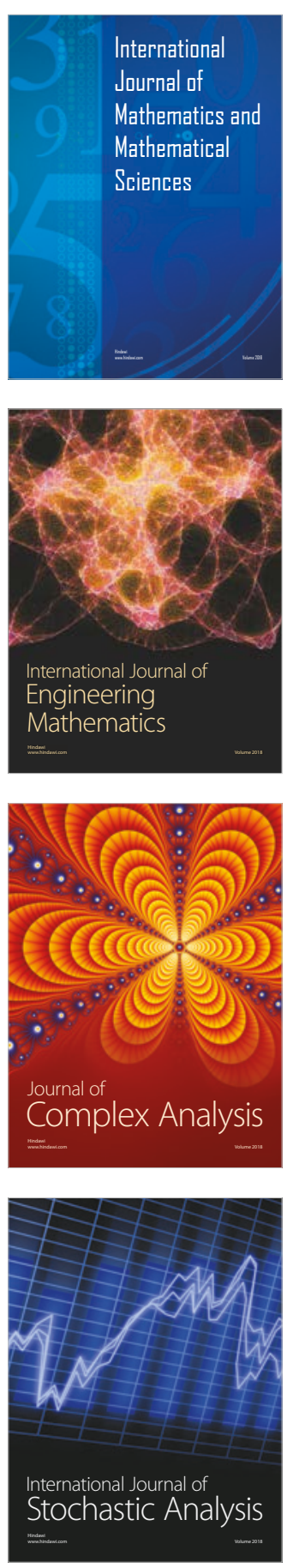
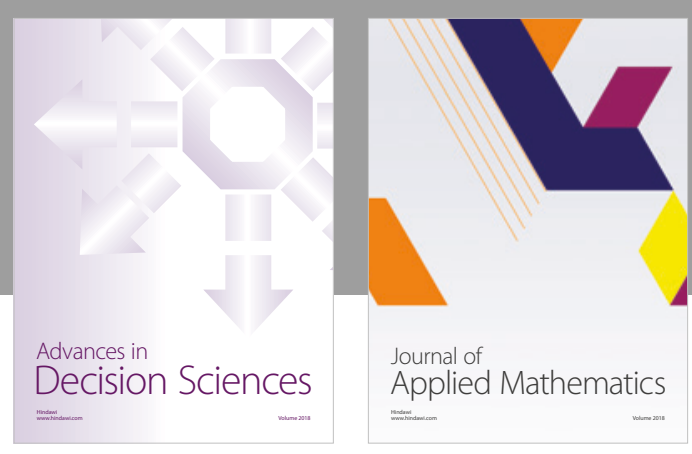

Journal of

Applied Mathematics
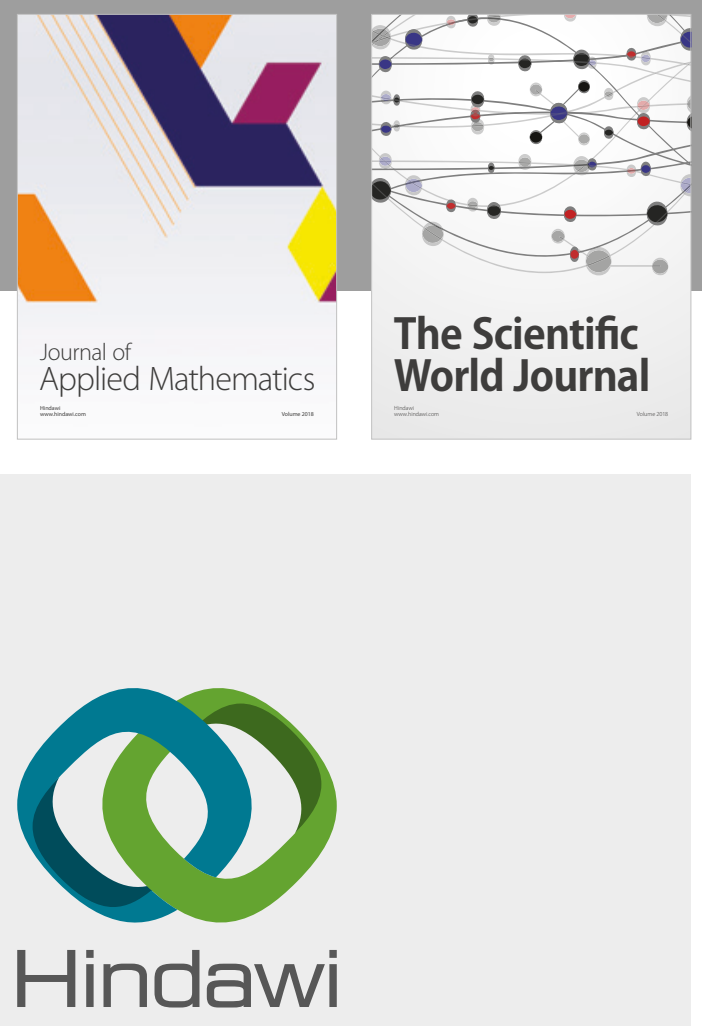

Submit your manuscripts at

www.hindawi.com

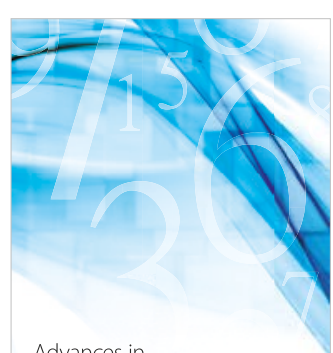

Advances in
Numerical Analysis
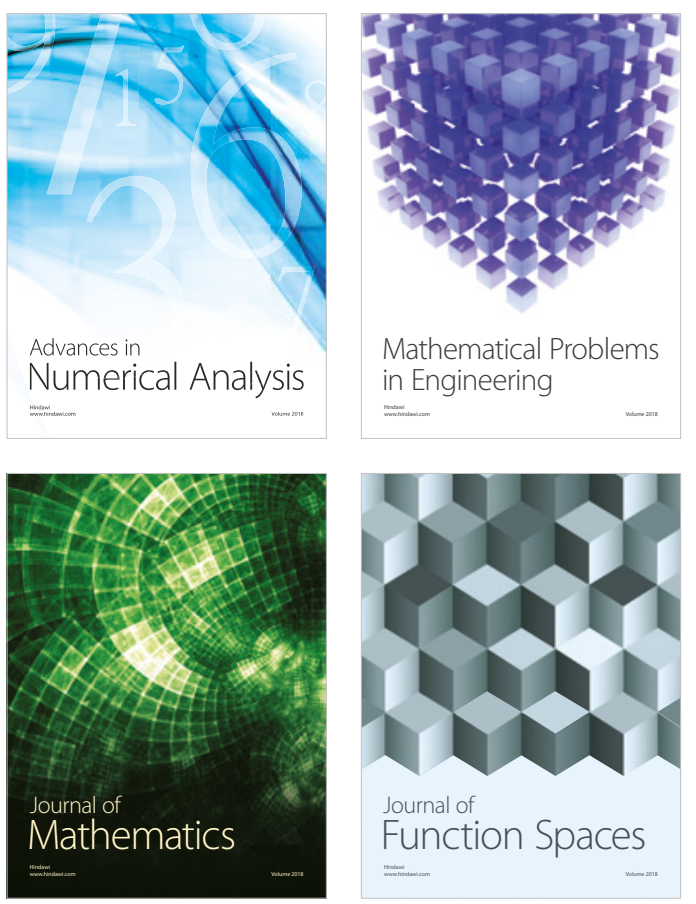

Mathematical Problems in Engineering

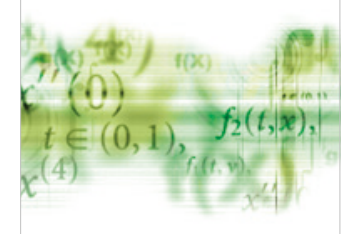

International Journal of

Differential Equations

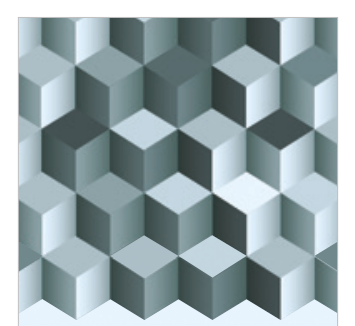

Journal of

Function Spaces

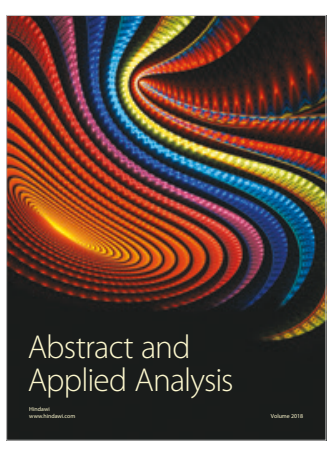

The Scientific

World Journal

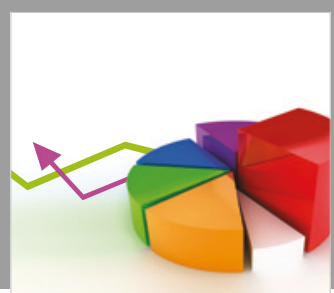

Journal of

Probability and Statistics
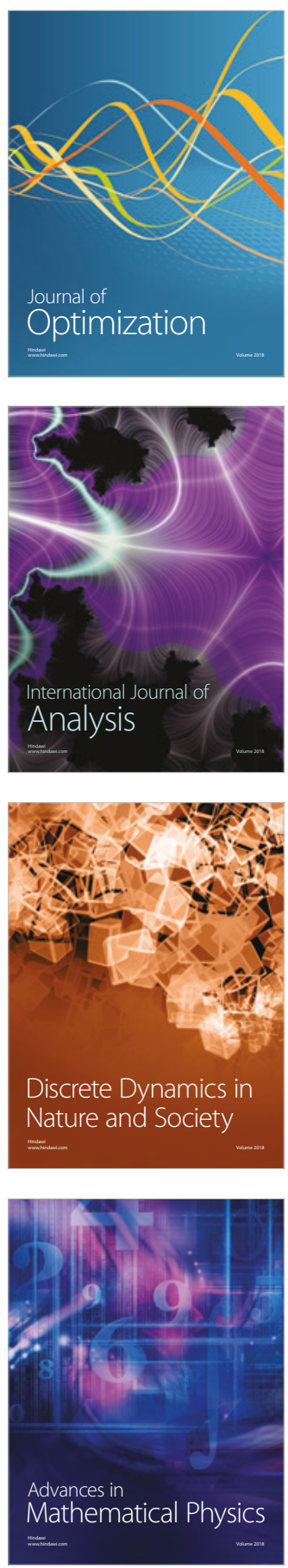